\title{
La investigación histórica como estrategia para la transformación de la enfermería
}

\section{Historical research as a Nursing transforming strategy}

Mtra. Edda Alatorre Wynter•

La auténtica investigación histórica implica filosofar. En efecto, no sólo es trabajo memorístico o anecdótico. Es un proceso para esclarecer las posibles relaciones entre diversos aconteceres y precisar sus causas y sus efectos.

No es mi objetivo detenerme a citar diversos conceptos sobre Historia, pero considero importante resaltar las coincidencias en que ésta tiene sentido si está al servicio del presente.

El pasado permite la mejor comprensión del presente cuando se sabe qué investigar, se posee claridad sobre los problemas históricos y, desde luego, cuando la investigación se apega con rigor a resolver los problemas metodológicos básicos: formulación de categorías conceptuales, fundamento de la periodización establecida, y rigor y confiabilidad en la crítica y análisis de fuentes. Asegurar el cumplimento de los aspectos metodológicos mencionados sólo nos lleva a la parte medular de la investigación: la interpretación de los hechos y su ubicación contextual.

Podemos hacer historia narrativa, anecdótica, anticuaria, cuando el interés se centra en el orden espacio-temporal de los acontecimientos pero sin establecer relaciones causales ni generalización alguna (efemérides).

Otro tipo de historia- reverencial, moralizante, conservadoracentra su interés en el registro de los acontecimientos que suelen celebrarse en las fiestas patrias, conmemoraciones, y cultos religiosos principalmente; busca la conservación de las instituciones oficiales gubernamentales, religiosas, educativas, etc. a través de la legitimación de: héroes y villanos, santos y demonios, mártires y verdugos.

En el lado opuesto de la historia anterior está la historia disruptiva, contestataria. Busca, más que explicaciones, desatarse del pasado, expulsar del presente las supervivencias que estorben a un proyecto particular. Indaga el lado sombrío del discurso oficial sobre los hechos. Se centra en la denuncia de las inequidades y de los mecanismos para conservarlas. Si bien este tipo de historia juega un papel importante en las luchas sociales, tiene el peligro de perder objetividad si no se cuida el rigor investigativo.

Finalmente, tenemos la historia científica, materialista, social. Constituye la ciencia de la historia. Se sustenta en las ciencias sociales: Sociología, Economía y Ciencia Política fundamentalmente. Aunque utiliza sobre todo métodos cualitativos tiene en la historia demográfica formas cuantitativas de investigación.

La historia científica, a través de una rigurosa investigación en fuentes históricas, busca la explicación racional de las relaciones sociales y con ello cuestiona la tendenciosa "naturalidad" de las desigualdades de clase y de género; descubre los intereses que conforman las fronteras geográficas, la jerarquización de clases y grupos, la aparición o extinción de instituciones y sus modalidades, la conformación del poder y del capital, la sexualización

कซै०

- Maestría en Enseñanza Superior y Diplomada en Estudios de Género y en Derechos Humanos.

Correspondencia: alatorre773@hotmail.com 
de las actividades humanas, la jerarquización de las profesiones y su fondo político etcétera.

Hacer una lectura histórica así nos permite tener una línea argumental para explicar lo social a partir del peso de lo simbólico en la materialidad de la existencia de las personas históricas hombres y mujeres.

En el caso concreto de la investigación histórica sobre la Enfermería-provisionalmente entendida ésta como las actividades de salud que históricamente ha realizado un grupo social específico: las enfermeras y los enfermeros- importará más que conocer fechas o relatos, conocer a los personajes que vivieron esa historia, quiénes eran, de qué estrato social provenían, qué hacían, porqué lo hacían, cómo lo hacían, cómo lo aprendían, qué lugar social les era asignado, qué relaciones de poder o de dependencia establecían unos y otras, qué se les permitía, qué se les prohibía, qué se les premiaba, qué se les sancionaba... Investigar así la historia de la Enfermería implica mucho más que una búsqueda documental, aún impecable, implica un largo camino de análisis, de reflexión, de argumentación, de construcción teórica, procesos mucho más complejos pero evidentemente mucho más útiles y significativos para entender el presente y construir el futuro de la Enfermería mexicana.

El análisis histórico-social de las profesiones constituye un espacio importante de investigación cuando se buscan los factores que expliquen su desarrollo e impulsen su avance y legitimación social.

Si bien cada profesión tiene particularidades propias, hay algunos eventos comunes que explican en mayor o menor medida su desarrollo, por ejemplo: su antigüedad, ${ }^{1}$ las condiciones sociales, económicas y políticas que le dieron origen, la naturaleza y legitimidad de su marco teórico referencial, las estructuras de poder en las que tuvo y tiene existencia y validez su formación y su práctica, y el grado de autonomía profesional de sus integrantes para ejercer un poder propio.

Aunque la investigación histórica puede hacerse desde diversos enfoques lo que la distingue es su intencionalidad. Historia para conservar o historia para transformar. Si lo que pretendemos es que la Enfermería se transforme, se desarrolle, que construya sus teorías, que empodere a las enfermeras en tanto mujeres-, que cuestione y trascienda el carácter femenino adjudicado arbitrariamente a la Enfermería y que ha dejado endeble la base legítima de los hombres que deseen ser enfermeros; que promueva una mejor prestación de servicios de salud; que aun en condiciones de interdisciplinariedad distinga su hacer específico en la docencia, la investigación, la práctica clínica, la administración; que sus integrantes ejerzan efectivamente sus derechos laborales y políticos; entonces, la investigación histórica de la Enfermería debe tener la intencionalidad de transformar, es decir, de producir los conocimientos que pongan en crisis las versiones ritualizadas del pasado. Allí, en su pasado, encontraremos la génesis de los mecanismos que explican el sistema de relaciones sociales jerarquizado y sexualizado en el que se sustenta la Enfermería de hoy.

Este enfoque aborda la investigación histórica sobre la base de las y los sujetos intervinientes, es decir desde una perspectiva de género, desde ésta, entendemos que toda situación social es resultado de un proceso de construcciones y de relaciones entre personas naturalmente diferentes - hombres y mujeres (sexo biológico)- pero construidas socialmente (género) como desiguales en detrimento de las mujeres. En este sentido, profundizar en la presencia histórica de las mujeres que se han desempeñado como enfermeras, en tanto sujetos históricos, hará visible el sistema que hace posible la conservación de una profesión sexualizada en el sentido de que aunque sea libre el ingreso para varones aún sigue viéndose "más normal" que la estudien sólo mujeres, por tanto, a pesar de pertenecer ellos al género

\footnotetext{
1. La antigüedad de una institución está dada por los eventos que sus integrantes consideran determinantes para el inicio de su presencia e importancia social. La UNAM celebró el pasado 22 de sep. el centenario de su fundación, aunque reconoce como antecedente cuatro y medio siglos en que se instituyó la Real y Pontificia Universidad de México, declara que es hasta hace cien años que inicia su rol definitivo como uno de los pilares institucionales del país. En el caso de la ENEO considero que 1945 es la fecha en que se inicia como institución, al dejar de ser una sección de la escuela de Medicina como venía siendo desde 1911 (año en que la escuela de Enfermería del Hospital General que le antecede desde 1907 pasa a Medicina) En esa fecha, ya con autonomía administrativa, es denominada con el nombre que aún conserva. Los eventos anteriores, en mi opinión, son antecedentes. A menos que nos parezca lógico que la ENEO tenga más antigüedad que la misma UNAM
} 
con más privilegios, están en una profesión donde arbitrariamente se les considera intrusos.

Desde esta perspectiva epistemológica estaremos en el terreno de transformar con teoría histórica la Enfermería y construirla sin limitaciones, para ser ejercida por personas femeninas y masculinas en igualdad de oportunidades y de poder.

La historia de las mujeres en el pasado es difícil de rastrear. ${ }^{2}$ Los datos que se tienen por lo general han sido escritos por hombres y con la visión estereotipada del "modelo ideal" de mujer obediente, abnegada, fiel, sumisa; o por el contrario: falsa, libertina, peligrosa, cuando no se ajustaba al modelo esperado.

Así también la historia de las enfermeras en el pasado es difícil de rastrear. Las razones son las mismas. La historia que hemos conocido ha sido más de tipo anecdótica y/o reverencial y en mi opinión ha contribuido en gran medida a tender una cortina de humo para no cuestionar el "orden" estructural en el que se reproducen las relaciones del poder médico institucional.

No se trata de escribir ahora una historia disruptiva y maniquea sobre lo que consideremos avances o retrocesos de la Enfermería como institución, sino incluir de manera paralela la historia social y política de sus integrantes; que no sólo busca reivindicar la historia de las mayorías- mujeres enfermerassino también y sobre todo, refor- mular los planteamientos teórico metodológicos de la ciencia histórica aplicada a la interpretación de la Enfermería, vista ésta como el conjunto de las experiencias de las mujeres y de los hombres en el pasado, que desde distintos espacios y relaciones de poder han escrito una parte importante de la historia de la atención de la salud en México.

Puesto que la Enfermería, sobre todo en el pasado, ha sido ejercida en su gran mayoría por mujeres, una estrategia metodológica es recurrir a la historiografía de las mujeres, especialidad que cada día cobra más impulso en la medida en que se buscan explicaciones más objetivas y racionales sobre la totalidad de los sujetos históricos. ${ }^{1-3}$

En este sentido, pocos espacios de análisis histórico brindan tanta necesidad de replanteamientos teórico metodológicos como la Enfermería, pues ha sido un espacio donde se ha mantenido, en mayor o menor medida, la identificación, la aceptación, y la actuación consecuente del "modelo enfermera" que complementa la construcción simbólica "médico/enfermera", en un sistema androcéntrico de atención de la salud.

Desde esta perspectiva, se convierte en oportunidades para la reflexión, para el análisis crítico y para proponer nuevos fundamentos teóricos a fin de trascender las estructuras que mantienen y reproducen la condición social de los y las enfermeras, deconstruyendo los

2 Celia Amorós (1991:27) señala que "la ausencia de la mujer en el discurso androcéntrico, como toda ausencia sistemática, es difícil de rastrear. Es la ausencia que ni siquiera puede ser detectada como ausencia porque ni siquiera su lugar vacío se encuentra en ninguna parte; es la ausencia de la ausencia

mecanismos ideológicos que han legitimado los atributos- lo que tienen que ser y lo que no-, los comportamientos- lo que deben ser y lo que no-, y los espacios- donde deben estar y donde no- diferenciados y jerarquizados para las personas, hombres y mujeres, que históricamente, informal y formalmente han cuidado, curado y rehabilitado en el campo de la salud.

Los atributos, los comportamientos y los espacios han estado y están sustentados en formas asimétricas de poder. La tarea es su deconstrucción y una estrategia para lograrlo es a través del análisis histórico social de la Enfermería en México y en el mundo pero con un enfoque de género, categoría de análisis construida desde la teoría social del feminismo que plantea que la realidad social es tal- sí y sólo sí- incluye por igual a mujeres y a hombres en todas las tareas que deciden el rumbo de la humanidad.

Los actores históricos del ejercicio de la Enfermería son sujetos en lo individual y de manera colectiva de una historia propia, compleja, diversa, que solo podrá conocerse con profundidad mediante un análisis que, sin pasar por alto su especificidad, los vincule con los procesos históricos económicos, políticos y sociales globales.

Un conocimiento histórico centrado en la Enfermería exige planteamientos innovadores, pero con el sustento metodológico de la disciplina histórica. La originalidad debe centrarse en el tipo de preguntas que se formulen a fin de hacer visibles a las personas que han transitado por la Enfermería en México, es decir, se trata de analizar la racionalidad histórica de la profesión en el contexto mexica- 
no y vincularla con el desarrollo de esta disciplina en otros contextos globales.

Se busca entonces la construcción de teoría particular y general en grandes temáticas, por ejemplo: ¿cuáles han sido las tendencias históricas que han prevalecido en la formación y en el ejercicio disciplinar y su funcionalidad social específica?, ¿cuáles han sido y son los principales problemas, contradicciones $\mathrm{y} / \mathrm{o}$ conflictos de la profesión y/o de sus egresados?, ¿cuál ha sido históricamente el mercado de trabajo?, ¿cuáles han sido y son y cómo se construyen las estructuras de poder formal?, ¿cómo y cuáles formas de poder y resistencia alternativas se han desarrollado?, ¿qué contradicciones han enfrentado y enfrentan las y los enfermeros entre la formación académica recibida y las necesidades laborales de las instituciones empleadoras y qué adecuaciones han hecho y hacen al respecto estas instituciones?, ¿cómo viven la formación y el ejercicio de la Enfermería los enfermeros en relación con las enfermeras?, ¿podemos identificar particularidades en el desarrollo profesional de unos y otras que sean atribuibles a su condición social de género?, ¿qué formas de poder legítimo y/o delegado identificamos en Enfermería?, ¿cómo se ejerce el poder en Enfermería?, ¿la formación académica ha sido y es congruente con egresar personas críticas y transformadoras y cómo se ha evaluado esto?. Por ejemplo.

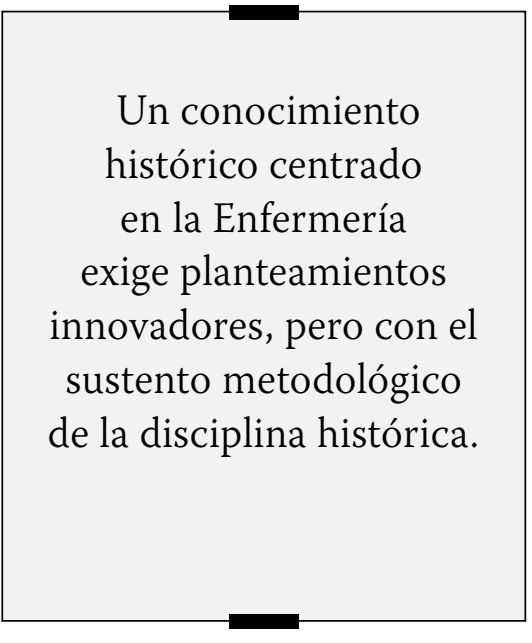

Para estudiar la Enfermería profesional, en principio conviene distinguir entre las actividades de dar atención a las personas enfermas -que ciertamente han sido ejercidas fundamentalmente por las mujeres por razones sociales y culturales- y las actividades no familiares o laborales en las que se establece un convenio entre quien otorga el servicio y quien lo requiere. Estas últimas actividades pueden ser no profesionales cuando carecen de educación formal o ésta no reúne los requisitos que la acrediten como profesión, y profesionales cuando cumplen con los lineamientos que legitiman académica y legalmente su ejercicio.

En México, la atención de los enfermos- como actividad ya laboral pero aún no formal- la encontramos en las épocas colonial e independiente y fue demandada en instituciones, primero de carácter religioso y más tarde laico, a hombres y mujeres por igual. No hay en un principio más jerarquía que la que otorgaba la posición social de

3 Curaban o cuidaban ¿quién hacía qué?. Me parece que en las palabras "curar y cuidar" hay más que meras diferencias de verbo. Ha una diferencia sustantiva de poder y esta diferencia puede ser el problema político que subyace en el quehacer de la Medicina y de la Enfermería

los participantes y la intencionalidad del servicio. ${ }^{4}$

El proceso de medicalización de la salud y el cambio en el modelo de atención durante el siglo $\mathrm{xx}$, profundiza y evidencia las asimetrías de poder en las tareas asistenciales. Se define el carácter androcéntrico de la medicina oficial y se sexualizan los saberes y los haceres en el proceso salud-enfermedad, es decir, se establece quién debía curar y quién cuidar. ${ }^{3}$

Desde el poder médico hegemónico se limita la participación masculina en tareas de Enfermería y se asegura un trabajo femenino de ayudantía a través de la formación y la ideologización de las mujeres enfermeras. Se institucionaliza este proceso con la creación de escuelas de Enfermería en el corto plazo y la extinción de las parteras universitarias, tiempo después. ${ }^{5}$

El análisis histórico social de la Enfermería en México es una tarea apasionante por la riqueza de eventos y la singularidad de sus personajes, mujeres- en su gran mayoría- y hombres luchando por hacer visible su historia en una estructura médica oficial, cuya naturaleza y poder explica el silencio de cualquier historia que no sea la propia.

En una primera aproximación, por ejemplo, a la Enfermería del siglo $\mathrm{xx}$, relacionando algunos eventos estructurales del modelo de atención médica con hechos de la historia de nuestra profesión, podemos partir de una periodización tentativa, por etapas:

La primera etapa (hasta la década de los cuarentas) se relaciona con las políticas de secularización, tecnificación y modernización de la asistencia médica. Se aprecia en México un desarrollo institucional 
rápido, un cambio en el modelo de atención médica, antes más particular y familiar a otro de carácter institucional y cautivo, y un incremento en la oferta de escuelas de medicina ante la demanda de recursos médicos para los, cada vez más numerosos, hospitales. Estas modificaciones en el modelo de atención cambian en consecuencia el modelo de atención de la Enfermería. Se requiere que quienes atiendan enfermos tengan mayor capacitación, lo que incrementa el nivel académico al ingreso de la naciente carrera, se multiplican las escuelas de Enfermería en México, los llamados a las mujeres para ser enfermeras y las limitaciones para que los hombres lo sean.

La segunda etapa está relacionada con el desarrollo de la salud pública después de la primera mitad del siglo xx. Se afianza la medicina institucional y se desarrollan las especialidades médicas. Con ello se incrementa la participación de las enfermeras en haceres más complejos, unos ligados a la especialidad médica y otros, aún no exclusivos de la medicina como la anestesia y la fisioterapia, que ejercieron las enfermeras y que después irían quedando en manos de los médicos. El Estado modifica sus políticas de salud hacia la Seguridad Social como modelo de atención, se crean las principales instituciones de salud (IMSS, ISSSTE), la Escuela de Medicina se convierte en facultad (1960), se inicia la crisis por sobrepoblación de recursos médicos al perderse la correspondencia entre la oferta y la demanda, pues al frenarse la expansión hospitalaria y tenerse completas las plazas médicas, no hay ya cabida para un número cada vez mayor de egresados, producto del incremento de escuelas de medicina en etapas anteriores. La Enfermería, en cuya formación y ejercicio se mantiene la visión médica, sigue orientando su práctica a la satisfacción de una ayudantía cada vez más eficiente.

La tercera etapa está relacionada con la masificación de las universidades- después de la década de los setentas. Grandes masas de estudiantes bachilleres buscan ingresar a éstas para asegurar movilidad social, se afectan los filtros para el ingreso con el llamado "pase automático", estrategia lograda por diversos grupos de presión y se dispara la matrícula. La inexistencia de un proyecto de planificación profesional y la libertad para elegir carrera ocasiona que las profesiones socialmente apreciadas como "masculinas" se saturen, y que las menos aceptadas o "femeninas"Enfermería entre ellas- dispongan de espacios, lo que lleva más adelante a reubicar a alumnos hombres a estas últimas con el consiguiente desánimo y frustración para ellos. La sobrepoblación médica y las insuficientes plazas laborales, aunado a los recortes presupuestales como consecuencia de la crisis económi$\mathrm{ca}$, ocasiona un proceso de proletarización médica caracterizado por: mayores exigencias académicas para poder competir en el mercado de trabajo, desempleo y subutilización de estos profesionistas, y ubicación en áreas de trabajo, otrora de Enfermería. Se inicia la Enfermería profesional en México. ${ }^{4}$

La Enfermería en la última década del siglo $\mathrm{xx}$ y en la primera del siglo XXI, ha venido centrando su interés en la construcción de nuevos paradigmas: por un lado en una práctica independiente sustentada en proyectos gerenciales y por otro lado, en una Enfermería institucional donde el destinatario de sus servicios no sea el médico sino el usuario y su familia. Hay un gran interés en la salud como derecho humano y en el cuidado como base del ejercicio profesional. ${ }^{5}$

La historia de la Enfermería es en gran medida la historia de las mujeres y hoy éstas, están luchando por cuestionar la supuesta naturalidad de las desigualdades sociales que viven; también en Enfermería el reto es cuestionar muchas desigualdades profesionales con respecto de otras profesiones.

Evidenciarlas y trascenderlas, proponer paradigmas alternativos no sólo ajustes y matices a la formación y al ejercicio profesional es tarea para las enfermeras y los enfermeros de hoy. Construir una Enfermería sin los estereotipos de género que históricamente le han atribuido y que, aunque se diga que ya no están presentes, no pasará de ser retórica bien intencionada mientras los discursos no se traduzcan en acciones académicas y políticas consecuentes, que sólo pueden edificarse sobre la base de evidencias históricas.

Pretender formar profesionales críticos, transformadores, significa

\footnotetext{
4 Si bien tiene una etapa antecedente con la apertura de la primera escuela de Enfermería en 1907, es profesión hasta que cumple con los requisitos académicos y sus egresados obtienen el grado de licenciados (as). El registro de títulos profesionales con este nivel se inicia en la Dirección General de Profesiones en la década de los setentas. Aquí hay otro espacio para obtener evidencias históricas
} 
un enorme y maravilloso compromiso que debe empezar por mirar, desde otra perspectiva epistemológica, las estructuras de poder donde se produce y se reproduce el conocimiento, donde se forman los actores de la política y el poder. Esto puede ser la diferencia entre egresar enfermeras y enfermeros conscientes, seguros, empoderados, que realmente actúen con un fundamental sentido social y humanístico incluyente. $\mathrm{O}$ por el contrario, egresar profesionales con licenciatura, con maestría y con doctorado en Enfermería, pero que aún enfrentan sin solución problemas ya tan añejos que seguirán siendo invisibles mientras no hagamos visibles los estereotipos de género impuestos a las tareas asistenciales y la asimetría de poder que subyace.

El feminismo concibe una humanidad incluyente. La investigación histórica desde la perspectiva de género, será seguramente una herramienta para construir una Enfermería incluyente. Las enfermeras y los enfermeros debemos proponer una Enfermería que no reproduzca en su interior la educación y el trabajo sexualizado, donde la diversidad no signifique desigualdad, en ningún espacio de formación o de práctica profe- sional, ni privilegios por razón de género. Una Enfermería construida por personas femeninas y masculinas igualmente diferentes.

Estoy consciente de que es una tarea muy compleja y a largo plazo, que debe nutrirse de la visión y las experiencias de las enfermeras y los enfermeros interesados en hacer posible esta utopía.

\section{Referencias Bibliográficas}

1 Ramos C. Introducciòn, En: RamosEscandón C (editora) Género e Historia, la historiografía sobre la mujer. México: Instituto Mora; 1992

2 Asunción Lavrin, La Mujer en México: 20 años de estudio, 1968-1988, ensayo historiográfico. En: Memorias del Simposio de Historiografía Mexicanista; 1988, octubre 11-14; Oaxtepec, México, Comité Mexicano de Ciencias Históricas IIH, 1990; pp. 545-80.

3 García AL. Problemas metodológicos de la historia de las mujeres: la historiografía dedicada al siglo XIX Mexicano. México: UNAMCoordinación de HumanidadesPUEG; 1994.

4. Alatorre-Wynter E. Hacia un nuevo perfil académico profesional en la Escuela Nacional de Enfermería y Obstetricia de la UNAM desde la

5 La construcción y la resignificación del conocimiento sobre la salud y el cuidado tienen que ver con relaciones de poder. En este contexto, la medicina oficial descalifica cualquier conocimiento ( como el cuidado ) si éste problematiza su base de poder. Me parece comprensible que, por ejemplo, el Dr. F. Fernández del Castillo (1953) señalara que "si por enfermera entendemos a la mujer que cuida a los enfermos, podemos decir que su profesión es tan antigua casi como la humanidad en la tierra". Pero que aún hoy las y los enfermeros lo sigamos diciendo es preocupante. Que el cuidado sea tan antiguo y connatural a las mujeres es un discurso funcional al poder médico. En esa lógica ¿para qué tanto estudio y reivindicaciones laborales? Si cualquier mujer, con y sin títulos y grados, puede cuidar. Esto constituye otro importante espacio para la investigación socio histórica de la Enfermería al servicio de la resignificación del Cuidado.

perspectiva de Género. [Tesis para obtener el grado de maestría]. México: Facultad de Filosofía y Letras UNAM, 2004.

5 Alatorre-Wynter E. Desarrollo histórico de la Enfermería en México. México: ENEO, UNAM; 1984.

\section{Bibliografía Recomendada}

Alatorre-Wynter E. Filosofía de la Educación en Enfermería. El caso de la ENEO de la UNAM. Maestría en Enseñanza Superior. Mecanograma 1991.

Alatorre-Wynter E. El proceso histórico de la educación de Enfermería en México. XIII Reunión Nacional de Licenciados en Enfermería. México: ENEO UNAM. 1992 pp. 49-67.

Alatorre-Wynter E. La salud de las mujeres, responsabilidad de las mujeres. México. Seminario "Presente y Prospectiva de la Mujer en Guanajuato". Guanajuato, Gto: FEMU. 1993.

Alatorre-Wynter E. La atención gineco-obstétrica en el marco de los Derechos Humanos. Memoria de la Reunión Nacional sobre Derechos Humanos de la Mujer. México: CNDH, 1995.

Alatorre-Wynter E. La Enfermería desde el enfoque del género. Revista Mexicana de Enfermería Cardiológica. 1996; 4(3): 84-7.

Alatorre-Wynter E, El 'deber ser' de las mujeres: una ojeada al pasado. Gaceta de la CNDH 1998; 92: 1828.

Alatorre-Wynter E. Antecedentes históricos de investigación de Enfermería en la ENEO. México: ENEO, UNAM Mecanograma. 1998.

Amorós C. Hacia una crítica de la razón patriarcal. 2a Edición Barcelona, España: Edit. Anthropos, 1991.

Castro R, Bronfman M. Teoría femi- 


\section{Enfermería Universitaria}

nista y Sociología Médica: Bases para una discusión. En: FigueroaPerea JG. (comp.). La condición de la mujer en el espacio de la salud. México: El Colegio de México; 1998 pp. 205-33.

Cortés R, Elvia M. et. al. Diagnóstico Social de la Profesión de Enfermería en México. Enfermería Hoy 1981; 1:11-62.

De Barbieri T. La desigualdad social a través del género. En: Fuentes $M$. Et.al. Ámbitos de familia. México: UNICEF, DIF, COLMEX; 1997. pp 85-8.

Defensoría del pueblo. Sobre género, derecho y discriminación. Lima, Perú: Pontificia Universidad Católica del Perú; 1999.

Ehrenreich B, English D. Inválidas o Contagiosas: Sexismo en el saber y la práctica médica. Revista Mujeres y Medicina; 1980: 1: 155-8.

Ehrenreich B, English D. Brujas, comadronas y enfermeras. Historia de las sanadoras. Barcelona: Edit. La Sal. 1984.

Facio A. Cuando el género suena cambios trae. Metodología para el análisis de género del fenómeno legal. San José, Costa Rica: ILANUD; 1992.

Fee E. Las mujeres y la atención a la salud: Una comparación de teorías. En: Cardaci, Revista Mujeres y Medicina; México: UNAM Xochimilco Archivo Histórico. 1987.
Fernández del Castillo F. Las facultades de Medicina según el Archivo de la Real y Pontificia Universidad de México. México: Edit. Imprenta Universitaria. 1953.

Flores-Palacios F. Psicología Social y Género. El sexo como objeto de representación social. México: DGAPA. UNAM. McGraw-Hill. 2000.

Foucault M. La arqueología del saber. México: Edit. Siglo XXI; 1979.

Foucault M. El nacimiento de la clínica. 18ª Ed. México: Edit. Siglo XXI. 1999.

Frenk J. El mercado de trabajo médico. México: UAM Xochimilco. 1980.

García AL. Problemas metodológicos de la historia de las mujeres: La historiografía dedicada al Siglo XIX mexicano. México: UNAM. Coordinación de Humanidades. PUEG., 1994.

García-Jiménez MA. García-Núñez M. (comp.) La Enfermería. México: UAM Xochimilco, 1991.

Gene L, Sue-Ellen E. Mujeres que trabajan en el Hospital. Revista Mujeres y Medicina. 1987: 3: 50-3.

González de León D. Las mujeres médicas. En: Cardaci D, González de León D (comps.) Las profesiones en México No. 3, México: Medicina. UAM Xochimilco. 1990.

González-Marín ML (coord.) Metodología para los estudios de Género. México: Edit. Instituto de Inves- tigaciones Económicas UNAM; 1996.

Gutiérrez-Castañeda G. Perspectiva de género: cruce de caminos y nuevas claves interpretativas. Ensayos sobre feminismo política y filosofía. México: UNAM Coordinación de Humanidades PUEG. 2002.

Heinrich-Kathleen T, Witt B. The passionate connection: Feminism Invigorates the Teaching of Nursing. Nurse Outlook 1993; 41(6): 117-24.

Lamas M. La antropología feminista y la categoría género. Rev. Nueva Antropología 1986; VIII (30): 173-97.

Lamas M. La perspectiva de género: una herramienta para construir equidad entre mujeres y hombres. México: DIF; 1997.

Pereyra C. Et.al. Historia ¿para qué?. 13a. Ed. México: Edit. Siglo XXI; 1990.

Pérez-Loredo L. Características de los servicios de Enfermería al inicio del siglo XX. XIII Reunión Nacional de licenciados en Enfermería. Memorias. México: ENEOUNAM. 1992.

Ríos-Everardo M. El género en la socialización profesional de enfermeras. México: UNAM.CRIM; 2001.

Scott JW. El problema de la invisibilidad. En: Ramos-Escandón C (editora) Género e historia: la historiografía sobre la mujer. México: Instituto Mora; 1992. 